Pacific Journal of Mathematics

IRREDUCIBLE CHARACTERS AND SOLVABILITY OF FINITE 


\title{
IRREDUCIBLE CHARACTERS AND SOLVABILITY OF FINITE GROUPS
}

\author{
F. R. DEMEYER
}

\begin{abstract}
The relationship between the degree of an irreducible character $\zeta$ on a finite group $G$ induced from a nilpotent normal subgroup and the structure of the group $G$ are studied when the degree of $\zeta$ is large. In particular if the square of the degree of $\zeta$ is the index of the center of $G$ in $G$ then $G$ is solvable.
\end{abstract}

Let $\zeta$ be an irreducible (complex) character on the finite group $G$. What conditions on $\zeta$ insure that $G$ is solvable? Of course, if $\zeta$ is a faithful linear character then $G$ is cyclic. We are interested in the other extreme when the degree of $\zeta$ is large, in part because of the relationship to the theory of projective representations and the Schur multiplier. Let $H$ be a nilpotent normal subgroup of $G$, assume $\zeta=$ $\phi^{G}$ for some character $\phi$ on $H$, and assume for each Sylow $p$-subgroup $S$ of $G$ that $\left.\zeta\right|_{S}=m \lambda$ for some irreducible character $\lambda$ on $S$ where $(m, p)=1$, then $G$ is solvable. If $Z$ is the center of $G$ the last condition always holds if the degree of $\zeta$ is $[G: Z]^{1 / 2}$, that is, if $G$ is a "group of central type" [2]. It is easy to see that no irreducible character on $G$ can have degree larger than $[G: Z]^{1 / 2}$. Another upper bound for the degree of an irreducible character on $G$ is $d[G: H]$ where $d=\max \{$ degree $\rho \mid \rho$ is an irreducible character on $H$ \} ([3] $17.9 \mathrm{p}$. 570). If $[G, G]$ is the commutator subgroup of $G$ and $Z \cap[G, G]$ contains an element of order $d[G: H]$ then there is an irreducible character $\zeta$ of degree $d[G: H]$ on $G$. Moreover, $\zeta=\phi^{G}$ for some character $\phi$ on $H$, and for each Sylow $p$-subgroup $S$ of $G,\left.\zeta\right|_{S}=\sum_{j=1}^{n} \lambda_{j}$ where the $\lambda_{j}$ are irreducible characters on $S$ with $\lambda_{j}(1)$ equal to the $p$-part of $\zeta(1)(j=1, \cdots, n)$. If $n=1$ for each prime $p$ dividing [G:1] then $G$ is solvable. An example showing the necessity of the hypothesis on $n$ is given. The conditions on the character $\zeta$ with respect to the Sylow subgroups $S$ of $G$ restrict the action of $G$ on $S$. To illustrate this we show $G$ is nilpotent if and only if for every Sylow subgroup $S$ of $G$ and every irreducible character $\chi$ on $G,\left.\chi\right|_{s}=$ $m \lambda$ for some irreducible character $\lambda$ on $S$.

In what follows all groups are finite and all characters and representations are taken over the complex numbers. If $n$ is an integer and $p$ is a prime integer we let $n_{p}$ denote the largest factor of $n$ which is a power of the prime $p$. Our standard reference is [3] and all unexplained terminology and notation coincides with [3]. 
THEOREM 1. Let $\zeta$ be an irreducible character on the group $G$ and let $H$ be a nilpotent normal subgroup of $G$. Assume

1. $\zeta=\phi^{G}$ for some character $\phi$ on $H$.

2. For each Sylow p-subgroup $S$ of $G,\left.\zeta\right|_{S}=m \lambda$ for some irreducible character $\lambda$ on $S$ where $(p, m)=1$. Then $G$ is solvable.

Proof. A theorem of P. Hall ([3] 1.10 p. 662) asserts that a group is solvable if every Sylow subgroup has a complement, this theorem will be applied to $G / H$. Let $p$ be a prime dividing [G: 1], let $P$ be the Sylow $p$-subgroup of $H$ and $S$ a Sylow $p$-subgroup of $G$. Since $P$ is a characteristic subgroup of $H, P$ is a normal subgroup of $G$ and $P \subseteq S$. By Clifford's Theorem ([3] 17.3 p. 565)

$$
\left.\zeta\right|_{P}=e\left(\rho_{1}+\cdots+\rho_{n}\right)
$$

where the $\rho_{i}$ are inequivalent irreducible characters on $P$ conjugate in $G$. We determine the number $n$. By hypothesis $2,\left.\zeta\right|_{P}=\left.m \lambda\right|_{P}$ so $\left.\lambda\right|_{P}=e / m\left(\rho_{1}+\cdots+\rho_{n}\right)$ and the $\rho_{i}$ are conjugate in $S$ by Clifford's Theorem. Now $\left(\phi,\left.\zeta\right|_{H}\right) \geqq 1$ so by relabeling we can say $\left(\rho_{1},\left.\phi\right|_{P}\right) \geqq 1$. We claim $\rho_{1}^{S}=\lambda$ so $e / m=1$ and $n=[S: P]$. To verify the claim hypothesis 2 says the $p$-part of $\zeta(1)$ is $\lambda(1)$. Also, $\left.\phi\right|_{P}=q \rho_{1}$ (since $H$ is nilpotent) where $\rho_{1}(1)$ is a power of $p$ so $\rho_{1}^{S}(1)$ divides the $p$-part of $\phi^{\alpha}(1)=\zeta(1)$. Since $\lambda$ is contained in $\rho_{1}^{S}$ this implies $\lambda=\rho_{1}^{S}$ verifying the claim.

Now $G$ acts on $\rho_{1} \cdots \rho_{n}$ by conjugation and the inertia group $H^{*}$ of the action of $G$ on $\rho_{1}$ has index $n=\lambda(1) / \rho_{1}(1)=[S: P]$. Also $H^{*}$ contains $H$ since $H$ is nilpotent so $H^{*} / H$ is a $p$-complement in $G / H$. The Theorem of P. Hall completes the proof.

We next give a sufficient condition that $\zeta$ satisfy condition 2 of Theorem 1. (See [2] Theorem 2).

Theorem 2. Let $\zeta$ be an irreducible character on $G$ and let $Z$ be the center of $G$. If $\zeta(1)^{2}=[G: Z]$ then for each Sylow p-subgroup $S$ of $G,\left.\zeta\right|_{S}=m \lambda$ for some irreducible character $\lambda$ on $S$ and $(p, m)=1$.

Proof. By Schur's lemma $\left.\zeta\right|_{Z}=\zeta(1) \psi$ where $\psi$ is a linear character on $Z$. Then by reciprocity $\left(\zeta, \psi^{G}\right)=\left(\left.\zeta\right|_{z}, \psi\right)=\zeta(1)$ so by counting degrees, $\zeta(1) \zeta=\psi^{G}$. Let $S$ be a Sylow $p$-subgroup of $G$ and let $R$ be the subgroup of $G$ generated by $Z$ and $S$. Let $\lambda$ be an irreducible character of $R$ contained in $\psi^{R}$. By Schur's lemma $\left.\lambda\right|_{S}$ remains irreducible because the elements of $Z$ are represented by Sclars. Since $\lambda$ is contained in $\psi^{R}, \lambda^{G}=m \zeta$ for some integer $m$. By counting degrees

$$
m=[G: R] \lambda(1) / \zeta(1)
$$


Since $\lambda$ is irreducible on $S, \lambda(1)=p^{a}$ for some $a,[G: R]$ is prime to $p$ since $R$ contains $S$. The $p$-part of $\zeta(1)^{2}$ is $[S: S \cap Z]$. Thus $\lambda(1)^{2}=$ $[S: S \cap Z]$ and $\left(\zeta, \lambda^{G}\right)=\left(\left.\zeta\right|_{R}, \lambda\right)=\left(\left.\zeta\right|_{s},\left.\lambda\right|_{S}\right)=[G: Z] / \lambda(1)^{2}$. Thus $\left.\zeta\right|_{S}=$ $m \lambda$ where $m$ is the largest divisor of $\zeta(1)$ prime to $p$. We combine the first two results to obtain.

COROLLARY 1. Let $\zeta$ be an irreducible character on the group $G$, and let $H$ be a nilpotent normal subgroup of $G$. Assume $\zeta=\phi^{G}$ for some character $\phi$ on $H$ and $\zeta(1)^{2}=[G: Z]$ where $Z$ is the center of $G$. Then $G$ is solvable.

The principal theorem of [1] is now an easy consequence of Corollary 1.

CoROLlaRy 2. Let $\zeta$ be an irreducible character on the finite group $G$, and let $A$ be an abelian normal subgroup of $G$. If $\zeta(1)^{2}=$ $[G: A]^{2}=[G . Z]$ where $Z$ is the center of $G$ then $G$ is solvable.

Proof. Let $\phi$ be a linear constitutent of $\left.\zeta\right|_{A}$. Then by reciprocity, $\zeta$ is a constitutent of $\phi^{G}$. But $\zeta(1)=\phi^{G}(1)=[G: A]$ so $\phi^{G}=\zeta$. By Corollary $1, G$ is solvable.

We now verify some of the hypothesis of Theorem 1 in another situation. We begin by summarizing basic results relating ordinary representations, projective representions, and the Schur Multiplier. Our nontrivial assertions are the contents of 23.3, p. 629 of [3]. Let $G$ be a finite group with center $Z$, assume $n$ is the exponent of $[G, G] \cap Z$ and let $\bar{G}=G / Z$. Write

$$
G=\bigcup_{g \in \bar{G}} Z R(g)
$$

where $R(g)$ is an element in $G$ corresponding to $g$. Then $R\left(g_{1}\right) R\left(g_{2}\right)=$ $A\left(g_{1}, g_{2}\right) R\left(g_{1} g_{2}\right)$ where $A\left(g_{1}, g_{2}\right) \in Z$. Let $a \in[G, G] \cap Z$ order $n$ and let $\theta$ be a linear character on $Z$ which is faithful on the cyclic group generated by $a$. Define a 2-cycle $\alpha$ on $\bar{G}$ by

$$
\alpha\left(g_{1}, g_{2}\right)=\theta\left(A\left(g_{1}, g_{2}\right)\right) \text {. }
$$

Let $K^{*}$ be the multiplicative group of the complex numbers. The element $\alpha$ represents in the Schur multipler $H^{2}\left(\bar{G}, K^{*}\right)$ has order $n$.

Form the projective group algebra $K \bar{G}_{\alpha}$ and let $M$ be a left $K \bar{G}_{\alpha^{-}}$ module. For each $g \in \bar{G}$, left multiplication by $g$ on $M$ induces a $K$ linear transformation $T(g)$ of $M$ and

$$
T\left(g_{1}\right) T\left(g_{2}\right)=\alpha\left(g_{1}, g_{2}\right) T\left(g_{1} g_{2}\right) \text {. }
$$

If $x \in G$ then $x=z_{1} R\left(g_{1}\right)$ where $z_{1} \in Z$ and $g_{1} \in \bar{G}$. Let left multiplica- 
tion by $x$ on $M$ be the linear transformation $T^{*}(x)=\theta\left(z_{1}\right) T\left(g_{1}\right)$. If $y=z_{2} R\left(g_{2}\right) \in G$ then

$$
x y=z_{1} z_{2} A\left(g_{1}, g_{2}\right) R\left(g_{1} g_{2}\right)
$$

and

$$
T^{*}(x) T^{*}(y)=\theta\left(z_{1}\right) T\left(g_{1}\right) \theta\left(z_{2}\right) T\left(g_{2}\right)=\theta\left(z_{1} z_{2}\right) \theta\left(A\left(g_{1}, g_{2}\right)\right) T\left(g_{1} g_{2}\right)=T^{*}(x y) .
$$

Thus $M$ can be viewed as a $K G$-module. Notice that $M$ is irreducible over $K G$ if and only if $M$ is irreducible over $K \bar{G}_{\alpha}$. Also, note that $\left.T^{*}\right|_{z}=T^{*}(1)$. This process can be reversed when $M$ is a $K G$-module giving the $G$ representation $T^{*}$ if $\left.T^{*}\right|_{Z}=T^{*}(1) \theta$ for the given linear character $\theta$ on $Z$. Define a linear character $\psi$ on $G$ by the equation $\psi(x)=\operatorname{det}\left(T^{*}(x)\right)$. Since $a \in[G, G], \psi(\alpha)=1$. But $\psi(a)=\theta(a)^{m}$ where $m=T^{*}(1)$ so $n$ divides $T^{*}(1)$.

Let $S$ be a Sylow $p$-subgroup of $G$ and $\bar{S}$ the natural image of $S$ in $\bar{G}$. The element the restriction of $\alpha$ to $\bar{S}$ represents in $H^{2}(\bar{S}$, $\left.K^{*}\right)$ is realized by the equation $\alpha\left(y_{1}, y_{2}\right)=\theta\left(A\left(y_{1}, y_{2}\right)\right)$ in the group $S Z$. By ([3] 16.21, p. 118) $\alpha$ represents an element whose order is $n_{p}$ in $H^{2}\left(\bar{S}, K^{*}\right)$. In the correspondence of ([3] 23.3, p. 629) this implies $\theta$ is faithful on a cyclic group of order $n_{p}$ in $[S, S] \cap Z$. Form the projective group algebra $K \bar{S}_{\alpha}$. Now $M$ can be viewed as a $K \bar{S}_{\alpha}$-module, let $M=M_{1} \oplus \cdots \oplus M_{k}$ where the $M_{i}$ are irreducible $K \bar{S}_{\alpha}$ modules. As above, each $M_{i}$ affords an ordinary representation $T_{i}{ }^{*}$ on $S Z$ which is irreducible. The restriction of $T_{i}^{*}$ to $S$ is also irreducible since each $T_{i}^{* *}$ restricted to $Z$ is $T_{i}^{*}(1) \theta$. Also, $\theta$ is faithful on a cyclic group of order $n_{p}$ in $[S, S] \cap Z$ so arguing as before $n_{p}$ must divide the degree of $T_{i}^{*}$.

LemMa 1. Let $G$ be a finite group with center $Z$, let $a \in[G, G] \cap$ $Z$ of order $n$, and let $\theta$ be a linear character on $Z$ faithful on the cyclic group generated by a. Then

(1) $\theta^{G}=\sum_{i=1}^{s} \zeta_{i}(1) \zeta_{i}$ where $n \mid \zeta_{i}(1)$ and the $\zeta_{i}$ are inequivalent irreducible characters of $G$.

(2) If $\zeta$ is an irreducible character on $G$ with $\left(0^{G}, \zeta\right) \geqq 1$ and $S$ is a Sylow p-subgroup of $G$ then $\left.\zeta\right|_{S}=\sum_{j=1}^{l} b_{j} \lambda_{j}$ where $n_{p} \mid \lambda_{j}(1)$, the $\lambda_{j}$ are inequivalent irreducible characters on $S$, and the $b_{j}$ are positive integers.

Proof. Let $\zeta$ be an irreducible character on $G$. By Schur's lemma $\left.\zeta\right|_{Z}=\zeta(1) \psi$ for a linear character $\psi$ on $Z$. Now $\left(\zeta, \psi^{a}\right)=$ $\left(\left.\zeta\right|_{z}, \psi\right)=\zeta(1)$. This shows $\theta^{i}=\sum_{i=1}^{s} \zeta_{i}(1) \zeta_{i}$ where the $\zeta_{i}$ are inequivalent irreducible characters of $G$. If $T_{i}$ is the representation affording $\zeta_{i}$ then $\operatorname{det} T_{i}$ is a linear character on $G$. Since $a \in[G, G], 1=$ 
$\operatorname{det}\left(T_{i}(a)\right)=\operatorname{det}\left[\theta(a) T_{i}(1)\right]=\theta(a)^{\zeta_{i}(1)}$. Therefore $n \mid \zeta_{i}(1)$.

To prove (2) we need the analysis which preceded the lemma. Let $T^{*}$ be the ordinary representation on $G$ which affords $\zeta$ and $T$ the corresponding projective representation on $\bar{G}$. In this situaion we showed $\left.T^{*}\right|_{s}=T_{1}^{*}+\cdots+T_{k}^{*}$ where the $T_{i}^{*}$ are irreducible and their degree are divisible by $n_{p}$. Let $\lambda_{1}, \cdots, \lambda_{l}$ be a full set of inequivalent characters afforded by the $T_{1}^{*}, \cdots, T_{k}^{*}$. Then $\left.\zeta\right|_{s}=$ $\sum_{i=1}^{l} b_{j} \lambda_{j}$ where $b_{j}$ is the multiplicity of $\lambda_{j}$ in $\left.\zeta\right|_{s}$ and $\lambda_{j}(1)$ is the degree of some $T_{i}^{*}$ and so is divisible by $n_{p}$. We can now prove

THEOREM 3. Let $G$ be group with center $Z$. Let $H$ be a normal nilpotent subgroup of $G$ and let $d=\max \{\rho(1) \mid \rho$ is an irreducible character of $H\}$. If $[G, G] \cap Z$ contains an element of order $d[G: H]$ then there is an irreducible character $\zeta$ on $G$ so that $\zeta=\phi^{G}$ for some character $\phi$ on $H$, and for each Sylow p-subgroup $S$ of $G,\left.\zeta\right|_{S}=\sum_{i=1}^{n} b_{i} \lambda_{i}$ where $\lambda_{i}(1)=\zeta(1)_{p}$. If $n=1$ for each $p$ then $G$ is solvable.

Proof. Let $n=d[G: H]$ and let $a \in[G, G] \cap Z$ of order $n$. Let $\theta$ be a linear character on $Z$ which is faithful on the cyclic group generated by $a$. By the first part of LEmMA 1

$$
\theta^{G}=\sum_{i=1}^{s} \zeta_{i}(1) \zeta_{i}
$$

where $n \mid \zeta_{i}(1)$ and the $\zeta_{i}$ are inequivalent irreducible characters of $G$. We will show each of the $\zeta_{i}$ satisfy the conclusion of the Theorem. By 17.9 p. 570 of [3], $n$ is the largest possible degree of an irreducible character on $G$ so $n=\zeta_{i}(1)(i=1, \cdots, s)$ and $H$ is a maximal nilpotent normal subgroup of $G$ so $Z \cong H$. Now $\theta^{G}(1)=[G: Z]$ so $[G: Z]=s n^{2}$ where $s$ is the number of inequivalent $\zeta_{i}$ in $\theta^{G}$. By Clifford's Theorem (17.3 p. 565, [3])

$$
\left.\zeta_{i}\right|_{H}=e\left(\phi_{1}^{i}+\cdots+\phi_{m}^{i}\right)
$$

where the $\phi_{i}^{i}(j=1, \cdots, m)$ are inequivalent irreducible characters on $H$ conjugate in $G$. Now $\zeta_{i}$ is a constitutent of $\left(\phi_{j}^{i}\right)^{G}$ and $\left(\phi_{j}^{i}\right)^{G}(1) \leqq$ $d[G: H]=\zeta_{i}(1)$ so for each $j, \phi_{j}^{i}(1)=d$ and $\left(\phi_{j}^{i}\right)^{G}=\zeta_{i}$. This verifies the first conclusion of Theorem 3 for each $i(i=1,2, \cdots, s)$.

Let $S$ be a Sylow $p$-subgroup of $G$. By the second part of Lemma 1,

$$
\left.\zeta_{i}\right|_{S}=\sum_{j=1}^{l} b_{j} \lambda_{j}^{i}
$$

where the $\lambda_{j}^{i}$ are inequivalent irreducible characters on $S$ and $n_{p}$ divides $\lambda_{j}^{i}(1)$. Since $H$ is nilpotent, $d_{p}=\max \{\gamma(1) \mid \gamma$ is an irreducible character on $P\}$. If $\lambda$ is an irreducible constitutent of $\left.\lambda_{j}^{i}\right|_{P}$ then 
$\gamma^{S}(1) \leqq \lambda_{j}^{i}(1)$ so $\gamma^{S}=\lambda_{j}^{i}$ and $\lambda_{j}^{i}(1)=d_{p}[S: P]=n_{p}$. This verifies the second conclusion of Theorem 3. If $n=1$ for each $p$ then $\left.\zeta\right|_{s}=b_{1} \lambda_{1}$ and $\zeta(1)=b_{1} \lambda_{1}(1)$. But $\zeta(1)_{p}=\lambda_{1}(1)_{p}$ so $\left(p, b_{1}\right)=1$ and by Theorem 1 , $G$ is solvable. This completes the proof.

For an example to show the necessity of Condition 2 in Theorem 1 let $H$ be any group of order $n$ and $J_{n}(H)$ the group algebra of $H$ over the ring $J_{n}$ of integers modulo $n$. Let $A=J_{n}(H)$ viewed as an additive group and let $H$ act as a group of automorphisms of $A$ by

$$
h(a x)=a h x \text { (regular representation) } x, h \in H, a \in J_{n} .
$$

Let $G$ be the semi-direct product of $A$ by $H$ with respect to this action. Let $\phi$ be the linear character defined on $A$ by $\phi\left(\sum_{h \in H} a_{h} h\right)=$ $\xi^{a}$ where $\xi$ is a primitive $n^{\text {th }}$ root of 1 and $a$ is an integer representing the coefficient in $J_{n}$ of the identity $e$ of $H$. One checks that $[G, A] \cap$ $Z=Z$ where $Z$, the center of $G$, is $\left\{\sum a_{h} h \mid a_{h}=a_{k}\right.$ all $\left.h, k \in H\right\}$ and has exponent $n$. Also $\phi$ is distinct from all its conjugates so $\phi^{G}=\zeta$ is irreducible. Yet $G$ need not be solvable. The problem is that the restriction of $\zeta$ to a Sylow subgroup does not behave properly. For example, if $H=A_{5}$ (the simple group of order 60), and $S$ is the Sylow 5-subgroup of $G$ then $\left.\zeta\right|_{S}=\sum_{i=1}^{12} \lambda_{i}$ where the $\lambda_{i}$ are 12 distinct irreducible characters on $S$ of degree 5 .

If $G$ is a finite group with center $Z$ and $\zeta$ is a faithful irreducible character on $G$ with $\left.\zeta\right|_{S}=m \lambda$ for some Sylow subgroup $S$ and irreducible character $\lambda$ on $S$ then the center of $S$ is $Z \cap S$. The proof of this observation also proves

THEOREM 4. The group $G$ is nilpotent if and only if for each irreducible character $\zeta$ on $G$ and each Sylow subgroup $S$ of $G,\left.\zeta\right|_{S}=$ $m \lambda$ for some irreducible character $\lambda$ on $S$.

Proof. Assume $G$ is nilpotent, let $\zeta$ be an irreducible character on $G$ and $S$ a Sylow subgroup. Then $S$ is normal in $G$ so by Clifford's Theorem

$$
\left.\zeta\right|_{S}=e\left(\phi_{1}+\cdots+\phi_{m}\right)
$$

with the $\phi_{i}$ distinct conjugate irreducible characters on $S$. If $g \in G$ then $g=g_{1} g_{2}$ where $g_{1}$ centralizes $S$ and $g_{2} \in S$. Then, $\phi_{1}^{g}=\phi_{1}^{g_{1} g_{2}}=$ $\phi^{g_{2}}=\phi$. So $m=1$.

Conversely, let $S$ be a Sylow subgroup of $G$ and let $\alpha$ be an element of the center of $S$. Let $\zeta$ be an irreducible character on $G$, then $\left.\zeta\right|_{S}=m \lambda$ where $\lambda$ is an irreducible character on $S$. Let $Z(S)$ be the center of $S$. Then by Schur's lemma, $\left.\lambda\right|_{Z(S)}=\lambda(1) \theta$ for some linear character on $Z(S)$. Thus $\zeta(a)=\zeta(1) \theta(a)$ so $a$ is an element of 
the center of $G / \operatorname{ker} \zeta$. Since this is true for all irreducible characters on $G$, a is an element of the center of $G$. If $\langle a\rangle$ is the central subgroup of $G$ generated by $a$ then the irreducible characters of $G /$ $\langle a\rangle$ correspond to the irreducible characters of $G$ with kernel $\langle a\rangle$. Thus $G /\langle a\rangle$ satisfies the same hypothesis $G$ does so by induction $G$ is nilpotent.

\section{REFERENCES}

1. Frank R. DeMeyer, Groups with an irreducible character of large degree are solvable, Proc. Amer. Math. Soc., 25 (1970), 615-617.

2. Frank R. DeMeyer and G. J. Janusz, Finite groups with an irreducible representation of large degree, Math. Zeit., 108 (1969), 145-153.

3. B. Huppert, Endliche Gruppen I, Springer-Verlag, Berlin-Heidelberg-New York, 1967.

Received March 19, 1971 and in revised form November 13, 1971.

Colorado State University 



\section{PACIFIC JOURNAL OF MATHEMATICS}

\section{EDITORS}

\author{
H. SAMELSON \\ Stanford University \\ Stanford, California 94305

\section{R. HoвBY} \\ University of Washington \\ Seattle, Washington 98105
}

\section{J. DugundJI}

Department of Mathematics University of Southern California Los Angeles, California 90007

\author{
RichaRd ARENS \\ University of California \\ Los Angeles, California 90024
}

\section{ASSOCIATE EDITORS}
E. F. BECKENBACH
B. H. NEUMANN
F. WOLF
K. YoshidA

\section{SUPPORTING INSTITUTIONS}

\author{
UNIVERSITY OF BRITISH COLUMBIA \\ CALIFORNIA INSTITUTE OF TECHNOLOGY \\ UNIVERSITY OF CALIFORNIA \\ MONTANA STATE UNIVERSITY \\ UNIVERSITY OF NEVADA \\ NEW MEXICO STATE UNIVERSITY \\ OREGON STATE UNIVERSITY \\ UNIVERSITY OF OREGON \\ OSAKA UNIVERSITY
}

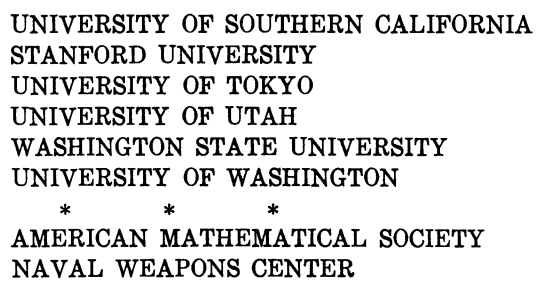

The Supporting Institutions listed above contribute to the cost of publication of this Journal, but they are not owners or publishers and have no responsibility for its content or policies.

Mathematical papers intended for publication in the Pacific Journal of Mathematics should be in typed form or offset-reproduced, (not dittoed), double spaced with large margins. Underline Greek letters in red, German in green, and script in blue. The first paragraph or two must be capable of being used separately as a synopsis of the entire paper. The editorial "we" must not be used in the synopsis, and items of the bibliography should not be cited there unless absolutely necessary, in which case they must be identified by author and Journal, rather than by item number. Manuscripts, in dup icate if possible, may be sent to any one of the four editors. Please classify according to the scheme of Math. Rev. Index to Vol. 39. All other communications to the editors should be addressed to the managing editor, Richard Arens, University of California, Los Angeles, California, 90024.

50 reprints are provided free for each article; additional copies may be obtained at cost in multiples of 50 .

The Pacific Journal of Mathematics is published monthly. Effective with Volume 16 the price per volume (3 numbers) is $\$ 8.00$; single issues, $\$ 3.00$. Special price for current issues to individual faculty members of supporting institutions and to individual members of the American Mathematical Society: $\$ 4.00$ per volume; single issues $\$ 1.50$. Back numbers are available.

Subscriptions, orders for back numbers, and changes of address should be sent to Pacific Journal of Mathematics, 103 Highland Boulevard, Berkeley, California, 94708.

PUBLISHED BY PACIFIC JOURNAL OF MATHEMATICS, A NON-PROFIT CORPORATION

Printed at Kokusai Bunken Insatsusha (International Academic Printing Co., Ltd.), 270, 3-chome Totsuka-cho, Shinjuku-ku, Tokyo 160, Japan. 


\section{Pacific Journal of Mathematics}

\section{Vol. 41, No. 2 December, 1972}

Tom M. (Mike) Apostol, Arithmetical properties of generalized Ramanujan sums .......................................... 281

David Lee Armacost and William Louis Armacost, On p-thetic groups ........ 295

Janet E. Mills, Regular semigroups which are extensions of groups .......... 303

Gregory Frank Bachelis, Homomorphisms of Banach algebras with minimal ideals ................................................ 307

John Allen Beachy, A generalization of injectivity .................. 313

David Geoffrey Cantor, On arithmetic properties of the Taylor series of rational functions. II.........................................

Václáv Chvátal and Frank Harary, Generalized Ramsey theory for graphs. III.

Small off-diagonal numbers .................................. 335

Frank Rimi DeMeyer, Irreducible characters and solvability of finite groups . . . . 347

Robert P. Dickinson, On right zero unions of commutative semigroups........ 355

John Dustin Donald, Non-openness and non-equidimensionality in algebraic

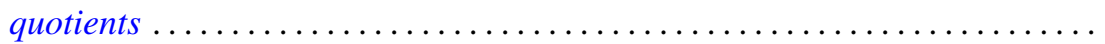

John D. Donaldson and Qazi Ibadur Rahman, Inequalities for polynomials with a prescribed zero ........................................ 375

Robert E. Hall, The translational hull of an $N$-semigroup ................ 379

John P. Holmes, Differentiable power-associative groupoids.............. 391

Steven Kenyon Ingram, Continuous dependence on parameters and boundary data for nonlinear two-point boundary value problems .

Robert Clarke James, Super-reflexive spaces with bases ..........

Gary Douglas Jones, The embedding of homeomorphisms of the plane in

continuous flows...............................

Mary Joel Jordan, Period $H$-semigroups and $t$-semisimple periodic

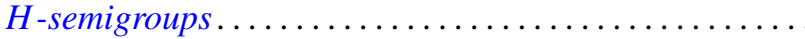

Ronald Allen Knight, Dynamical systems of characteristic 0

Kwangil Koh, On a representation of a strongly harmonic ring by sheaves...

Hui-Hsiung Kuo, Stochastic integrals in abstract Wiener space. ..

Thomas Graham McLaughlin, Supersimple sets and the problem of extending a

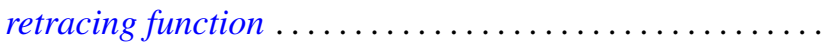

William Nathan, Open mappings on 2-manifolds .

M. J. O'Malley, Isomorphic power series rings

Sean B. O'Reilly, Completely adequate neighborhood systems and metrization

Qazi Ibadur Rahman, On the zeros of a polynomial and its derivative...

Russell Daniel Rupp, Jr., The Weierstrass excess function ..

Hugo Teufel, A note on second order differential inequalities and functional

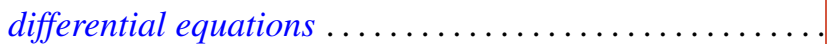

M. J. Wicks, A general solution of binary homogeneous equations over free 